\title{
Effect of Time of Cord Clamping on Serum Bilirubin Level among Full Term Babies Born at Tikur Anbessa Specialized Hospital: A Three Arm Randomized Control Trial.
}

Biruk Hailu Tesfaye ( $\square$ brkhailu41@gmail.com )

Addis Ababa University

Mulugeta Betre Gebremariam

Addis Ababa University

Abiy Seifu Estifanos

Addis Ababa University

Asrat D. Gebremedhin

Addis Ababa University

\section{Research Article}

Keywords: delayed cord clamping, serum bilirubin, newborns

Posted Date: February 24th, 2021

DOl: https://doi.org/10.21203/rs.3.rs-237034/v1

License: (a) (i) This work is licensed under a Creative Commons Attribution 4.0 International License.

Read Full License 


\section{Abstract}

Background: Delayed cord clamping (DCC) above one minute is associated with nutritional and developmental benefits newborns. DCC could also lead to elevated serum bilirubin, leading to jaundice that require phototherapy at the early ages of life. However, evidence on the relationship between DCC and outcomes is limited in resource-limited settings.

Objective: To evaluate the effect of the time of cord clamping on serum bilirubin level at 24 hours postbirth in an Ethiopian Tertiary Care hospital.

Methods: A three-arm, single-blind, randomized controlled trial was conducted between October 2019January 2020 targeting healthy, full-term neonates. Study participants were randomly assigned into one of the following interventions depending on the timing of cord clamping: immediate cord clamping ( $<30$ seconds), intermediate cord clamping (60 sec), and delayed cord clamping (180 seconds). The primary outcome is serum bilirubin level in the newborns, which was assessed at 24hour after birth and before they were discharged home. Descriptive analysis was applied to assess differences between groups in terms of demographic, obstetric, and social characteristics of participants, while linear regression model was employed to examine the association between cord clamping time and serum bilirubin level. STATA version 14 was used for statistical analyses.

Result: A total of 141 term newborns were included and randomized into the three categories. Demographic, social and obstetrics factors of the women were not different across groups except labor duration that had a P-value $<0.05$. Time of cord clamping was not a significant predictor of total serum bilirubin levels at 24 hours post-birth, whereas cord blood total serum bilirubin (coef. $0.24 ; p<0.05$ ) and bilirubin nomogram high-risk zone (Coef. $6.25 ; p<0.001$ ) were significant predictors.

Conclusion: Time of umbilical cord clamping has no effect on the total serum bilirubin level of neonates at least within 24 hours of birth.

\section{Introduction}

According to global estimates, Ethiopia is one of the top nine countries that contribute half of the total childbirth around the world with more than 9000 births every day. ${ }^{1}$ From the total live births, around $75 \%$ of them are delivered with spontaneous vaginal delivery under the risk of facing several challenges and complications around time of birth. ${ }^{2}$

Jaundice (hyperbilirubinemia) is one of the challenges within the first 24 hours of childbirth. Hyperbilirubinemia is defined as an excessive level of bilirubin ( $>34.2 \mu \mathrm{moL} / \mathrm{L}$ or $2 \mathrm{mg} / \mathrm{dL}$ ) either in the conjugated or unconjugated form. ${ }^{3}$ Eighty percent of bilirubin is derived from the heme group of hemoglobin, which comes from the destruction of red blood cells (RBCs) in the reticuloendothelial of the liver, spleen, and bone marrow. ${ }^{4}$ The destruction of RBCs could occur either due to premature rupture or 
excessive RBCs (polycythemia) and is directly influenced by the delay in the clamping time that gives extra $80-100 \mathrm{~mL}$ of blood within the first $1-3$ minutes to enter the neonate circulation. ${ }^{5-7}$

The case fatality rate for neonatal jaundice and severe complications in Low- and Middle-Income Countries range between $20 \%$ and $60 \% .{ }^{8}$ The case in Ethiopia is not different from the rest of Africa; it is estimated that about $13 \%$ of neonates born in hospitals develop neonatal jaundice, while $7 \%$ of them develop severe related complications like bilirubin encephalopathy. ${ }^{9}$

During the first few minutes after birth, there is a circulation of blood from the placenta to the infant. Delaying clamping of the umbilical cord by 2 to 3 minutes or until the pulsation of the cord stops will allow the physiological transfer of placental blood to the infant. ${ }^{3}$ Evidence shows that delayed cord clamping is beneficial in preventing neonatal hospital mortality. ${ }^{10}$ This is mainly because of its potential to lower the risk of reduced hematocrit level at 24 hours and also reducing the risk of iron deficiency at 36 months post-birth. ${ }^{10,11}$ The implementation of delayed cord clamping (DCC) has a good deal of support globally for the healthy outcome of neonates and mothers, despite arguments on its potential adverse effects.

Although multiple studies revealed the immediate and long-term benefits of implementing DCC for both the neonate and the mother, ${ }^{3}$ emerging anecdotal evidence reported the counter effect of delaying the cord clamping; mainly attributed to the increasing neonatal risk of polycythemia and neonatal jaundice that require phototherapy. ${ }^{11,12}$

Based on the findings from various randomized clinical trials and reviews, the World Health Organization (WHO) recommends delayed umbilical cord clamping (not earlier than 1 min after birth), as it can improve maternal and infant health and nutrition outcomes. ${ }^{3}$ However, there is a need for studies to establish the association between DCC and risk of hyperbilirubinemia on newborns. ${ }^{3}$ Particularly, there is a relative paucity of data from resource-limited settings on the clinical outcomes associated with the implementation of delayed clamping. Therefore, creating an evidence based on the effect of DCC on outcomes in newborns will be instrumental in informing clinical guidelines.

This study aims to evaluate the effect of time of umbilical cord clamping on serum bilirubin level and proportion of hyperbilirubinemia that requires phototherapy.

\section{Methods}

\section{Study setting}

This three-arm parallel randomized control trial was conducted at Tikur Anbessa Specialized hospital (TASH) -the largest referral and teaching hospital in Ethiopia. The study protocol (attached as appendix) was approved by the institutional review board at the College of Health Sciences, Addis Ababa University (AAU/SPH-IRB/0056) and respective departments of obstetrics/gynecology and neonatology with in the 
TASH. written informed consent was provided by the participants before they were randomized in the study.

\section{Study population and recruitment}

The study participants were pregnant women with fetuses that are scheduled for a spontaneous vaginal delivery and with an inclusion criterion of; a gestational age of $\geq 37$ weeks as diagnosed with first day of last menstrual period (LMP) or ultrasound and those with no gross complications related with the pregnancy and delivery, including mal-presentation, fetal distress, and congenital malformations. Women at greater risk of adverse effects due to delayed clamping were excluded from the study. The main exclusion requirements were those with previa or placenta abruption, early diagnosed fetal anomalies, those with fetal anemia, those with pre-eclampsia or significant maternal anemia. ${ }^{13}$ Participants were recruited in the period between October 2019 and January 2020.

\section{Randomization and interventions}

The eligible participants were initially approached by the principal investigator (who is a research midwife) to obtain consent before or on the day of delivery. The consented participants were randomized into three groups in a 1:1:1 ratio using pre-determined six block randomization sequences.

The interventions the different groups received are as follows:

Group 1: clamping of the umbilical cord immediately (<30seconds) after birth.

Group 2: clamping of the umbilical cord intermediately (30 - 60 seconds) after birth.

Group 3: clamping of the umbilical cord time was $\geq 180$ seconds (3-minutes) after birth.

The allocation of the participants was performed after obtaining their informed consent earlier in the late first stage labor (i.e. $\geq 8 \mathrm{~cm}$ cervical dilatation). The principal investigator $(\mathrm{PI})$ prepared the allocation codes with sealed, opaque, and identically colored envelopes initially at the beginning of the study. One of the co-investigators, who was blinded to the participant screening and randomization process, was responsible for opening the envelope and revealing the nature of intervention to the birth attendant when the mother enters the second stage of labor or was ready for childbirth proper.

\section{Outcomes}

The primary outcome is the level of total serum bilirubin of the newborns at the 24 hours of age and the secondary outcome is the proportion of neonates that developed hyperbilirubinemia before discharge to home.

\section{Data collection procedure}


The data collection had two phases. First, around time of birth; and both maternal and fetal characteristics were recorded. Second, at the age of 24 hour after delivery. Blood samples for the primary outcome were collected from veins and were transported to the laboratory with anti-coagulated test tube by one of the research investigators within 30 minutes of the sample collection. The source of sociodemographic and obstetric data was the medical record chart of both the mother and the newborn. The rest of the data was obtained from direct interview of the mother and laboratory findings. The sample size which was calculated using two independent proportions with a power of $90 \%$ and $a=0.05$ resulted in a total of 141 participants that were subsequently categorized into three arms ( 47 in each group).

The data collectors discussed with eligible participants and/or their companions about the procedure. The discussion includes explaining the risks and benefits associated with the interventions later in the first stage of labor. Informed consent was then secured either from the participant or respective companions before the second stage labor ensued or upon preparing the mother for delivery. When the mother is ready for delivery, the randomly assigned sealed-envelope containing the group assignment. The investigator monitored the clock with a digital second counter to inform the birth attendant when the time for that particular intervention ended.

After expulsion of the newborn, the birth attendant had to collect one milliliter cord blood and handed it to the assistant after separating the cord irrespective of the group assignment. The collected blood samples were then stored in a test tube, transported to the assigned laboratory. The results were recorded on the newborn chart by the attendant according to the hospital protocol.

\section{Statistical analysis and data management}

After data collection, the completeness and consistency of the questionnaires were checked. Then both the questionnaires and the variables coded, entered into the Epidata manager version 4.4.2.1 (Epi-Data Association, Denmark) and the necessary data cleaning performed.

Descriptive analysis was performed to compare baseline characteristics of the participants and reported using mean and standard deviation (SD) for discrete continuous data types. Ina addition, frequencies and percentages used for categorical data presentation. Pearson's chi-square test was used to compare categorical variables across the different cord clamping times, and Fisher's exact test used when there were less than five observations within the groups. Kruskal Wallis rank test was also used for comparison of continuous variables based on the time of clamping. We used unadjusted linear regression model to evaluate the effect of times for cord clamping on serum bilirubin level and multiple linear regression to conduct an adjusted analysis controlling for other important factors. Associations were considered significant at $p<0.05$ and analysis was performed using STATA version 14 software (Stata Corp LLC, College Station, TX, USA).

\section{Results}


Table 1 presents the basic demographic, social and obstetric characteristics of participant mothers. Most of the participants $(102 ; 72 \%)$ aged between $20-34$ years, while $(29 ; 20 \%)$ of them were under the age of 20 years. A total of $107(75.88 \%)$ participants have had the recommended antenatal visit of more than four times. From total participants, 127 (90.07\%) had normal and 14 (9.93\%) prolonged labor.

Table 1: Demographic and obstetric Summary characteristics of mothers gave birth at TASH, from October 2019 - January 2020

\begin{tabular}{|c|c|c|}
\hline & Frequency & Percentage \\
\hline \multicolumn{3}{|c|}{ Age of the mother } \\
\hline$<20$ & 29 & 20.57 \\
\hline $20-34$ & 102 & 72.34 \\
\hline$\geq 35$ & 10 & 7.09 \\
\hline \multicolumn{3}{|l|}{ Gravidity } \\
\hline Primiparous & 58 & 41.13 \\
\hline Multiparous & 83 & 58.87 \\
\hline \multicolumn{3}{|l|}{ ANC visits } \\
\hline$<4$ times & 34 & 24.12 \\
\hline$\geq 4$ times & 107 & 75.88 \\
\hline \multicolumn{3}{|c|}{ Labor Duration } \\
\hline Normal & 127 & 90.07 \\
\hline Prolonged & 14 & 9.93 \\
\hline \multicolumn{3}{|l|}{ Labor onset } \\
\hline Spontaneous & 120 & 85.11 \\
\hline Induced & 21 & 14.89 \\
\hline \multicolumn{3}{|l|}{ PROM } \\
\hline Yes & 19 & 13.48 \\
\hline No & 122 & 86.52 \\
\hline \multicolumn{3}{|c|}{ Caesarean section history } \\
\hline Yes & 15 & 10.64 \\
\hline No & 126 & 89.36 \\
\hline
\end{tabular}

The association between maternal and obstetrics factors were also compared based on the different time of umbilical cord clamping (Table 2). None of the factors showed a significant difference across the different cord clamping times except the duration of labor, which is significantly longer in the intermediate clamping time $(P$-value of $<0.05)$ compared with the immediate or delayed clamping groups. 
Table 2: Association between maternal and obstetric factors with time of umbilical cord clamping for mothers who gave birth at TASH from October 2019 - January 2020.

\begin{tabular}{|c|c|c|c|c|}
\hline Variables & Immediate clamping $(<30$ seconds $)(n=50)$ & $\begin{array}{c}30-60 \text { seconds } \\
(\mathrm{n}=45)\end{array}$ & $\begin{array}{c}\text { Delayed } \\
(>=180 \text { seconds }) \\
(\mathrm{n}=46)\end{array}$ & P-value \\
\hline \multicolumn{4}{|l|}{ Maternal Age - n (\%) } & 0.261 \\
\hline$<20$ & $11(22)$ & $10(22.2)$ & $8(17.4)$ & \\
\hline $20-34$ & $36(72)$ & $29(64.4)$ & $37(80.4)$ & \\
\hline$>=35$ & $3(6)$ & $6(13.4)$ & $1(2.2)$ & \\
\hline \multicolumn{4}{|l|}{ Gravidity - n (\%) } & \multirow[t]{3}{*}{0.629} \\
\hline Primiparous & $20(40)$ & $21(47)$ & $21(45.7)$ & \\
\hline Multiparous & $30(60)$ & $24(53)$ & $25(54.3)$ & \\
\hline \multicolumn{4}{|c|}{ Chronic Medical illness history - $\mathrm{n}(\%)$} & \multirow[t]{3}{*}{0.366} \\
\hline Yes & $8(16)$ & $3(66.7)$ & $6(13)$ & \\
\hline No & $42(84)$ & $42(33.3)$ & $40(87)$ & \\
\hline \multicolumn{4}{|l|}{ PROM - n (\%) } & \multirow[t]{3}{*}{0.800} \\
\hline Yes & $7(14)$ & $7(15.2)$ & $5(10.8)$ & \\
\hline No & $43(86)$ & $38(84.8)$ & $41(89.2)$ & \\
\hline \multicolumn{4}{|c|}{ Sibling history of jaundice $-\mathrm{n}(\%)$} & \multirow[t]{3}{*}{$0.309^{f e}$} \\
\hline Yes & $1(2)$ & $1(2.2)$ & $2(4.3)$ & \\
\hline No & $49(98)$ & $44(97.8)$ & $44(95.7)$ & \\
\hline \multicolumn{4}{|l|}{ CS history - n (\%) } & \multirow[t]{3}{*}{0.882} \\
\hline Yes & $6(12)$ & $4(8.9)$ & $5(10.9)$ & \\
\hline No & $44(88)$ & $41(91.1)$ & $41(89.1)$ & \\
\hline \multicolumn{4}{|c|}{ Labor Duration - n (\%) } & \multirow[t]{3}{*}{0.015} \\
\hline Normal & $46(92 \%)$ & $36(80)$ & $45(97.8)$ & \\
\hline Prolonged & $4(8 \%)$ & $9(20)$ & $1(2.2)$ & \\
\hline \multicolumn{4}{|l|}{ Labor onset - n (\%) } & \multirow[t]{3}{*}{0.092} \\
\hline Spontaneous & $45(90)$ & $34(75.5)$ & $41(89.1)$ & \\
\hline Induced & $5(10)$ & $11(24.5)$ & $5(10.9)$ & \\
\hline \multicolumn{4}{|c|}{ ABO incompatibility - n (\%) } & \multirow[t]{3}{*}{0.851} \\
\hline Yes & $4(8)$ & $5(11.1)$ & $5(10.9)$ & \\
\hline No & $46(92)$ & $40(88.9)$ & $41(89.1)$ & \\
\hline \multicolumn{4}{|c|}{$\mathrm{Rh}$ incompatibility - n (\%) } & \multirow[t]{3}{*}{0.527} \\
\hline Yes & $2(4)$ & $4(8.9)$ & $2(4.3)$ & \\
\hline No & $48(96)$ & $41(91.1)$ & $44(95.7)$ & \\
\hline \multicolumn{4}{|l|}{$\mathrm{PPH}-\mathrm{n}(\%)$} & \multirow[t]{3}{*}{0.593} \\
\hline Yes & $2(4)$ & $1(2.2)$ & $3(6.5)$ & \\
\hline No & $48(96)$ & $44(97.8)$ & $43(93.5)$ & \\
\hline fe-fisher's exact te & & & & \\
\hline
\end{tabular}

Table 3 presents the association between neonatal factors and time of cord clamping. The gender distribution is similar across the three groups. While most of the newborns $(94.3 \%)$ were fed with breastmilk only, this did not vary based on the time of cord clamping. APGAR score was also not significantly associated with the umbilical cord clamping time.

Majority of the newborns (95.74\%) were in the low-risk zone of the bilirubin nomogram within the first 24 hours of their birth and only three (2.12\%) newborns required phototherapy within 48-72 hours post-birth, albeit this was not significantly related with time of cord clamping. The mean weight of neonates during birth was $3251 \mathrm{gm}( \pm 60), 3259 \mathrm{gm}( \pm 63)$, and $3247 \mathrm{gm}( \pm 67)$ for immediate $(<30 \mathrm{sec})$, intermediate (30 $60 \mathrm{sec})$ and delayed $(\geq 180 \mathrm{sec})$ umbilical cord clamping times, respectively. Further, there were no variations in average temperature, and cord blood RBC and TSB between groups. 
Table 3: Association of neonatal factors with umbilical cord clamping time of newborns born at TASH between October 2019 January 2020.

\begin{tabular}{|c|c|c|c|c|}
\hline Variables & Immediate & $30-60$ seconds & Delayed & P-value \\
\hline \multicolumn{4}{|l|}{ Gender - n (\%) } & \multirow[t]{3}{*}{0.727} \\
\hline Male & $24(48 \%)$ & $25(56 \%)$ & $25(54 \%)$ & \\
\hline Female & $26(52 \%)$ & $20(44 \%)$ & $21(46 \%)$ & \\
\hline \multicolumn{4}{|l|}{ Type of Feeding - n (\%) } & \multirow[t]{4}{*}{$0.078^{f e}$} \\
\hline Breastmilk only & $48(96 \%)$ & $40(89 \%)$ & $45(98 \%)$ & \\
\hline Formula feeding & 0 & $5(11 \%)$ & 0 & \\
\hline Mixed Feeding & $2(4 \%)$ & 0 & $1(2 \%)$ & \\
\hline \multicolumn{4}{|c|}{$1^{\text {st }}$ minute APGAR Score $-\mathrm{n}(\%)$} & \multirow[t]{3}{*}{0.215} \\
\hline$<7$ & $12(24 \%)$ & $10(22 \%)$ & $5(11 \%)$ & \\
\hline$>=7$ & $38(76 \%)$ & $35(78 \%)$ & $41(89 \%)$ & \\
\hline \multicolumn{4}{|c|}{$5^{\text {th }}$ minute APGAR Score $-\mathrm{n}(\%)$} & \multirow[t]{3}{*}{$0.69^{f e}$} \\
\hline$<7$ & $1(2 \%)$ & $3(7 \%)$ & $1(2 \%)$ & \\
\hline$>=7$ & $49(98 \%)$ & $42(93 \%)$ & $45(98 \%)$ & \\
\hline \multicolumn{4}{|c|}{ Bilirubin nomogram risk zone - n (\%) } & \multirow[t]{4}{*}{$1.00^{f e}$} \\
\hline Low risk zone & $46(92 \%)$ & $45(100 \%)$ & $44(96 \%)$ & \\
\hline Low-intermediate risk zone & $1(4 \%)$ & 0 & $1(2 \%)$ & \\
\hline High risk zone & $1(4 \%)$ & 0 & $1(2 \%)$ & \\
\hline \multicolumn{4}{|l|}{ Phototherapy } & \multirow[t]{3}{*}{$1.00^{f e}$} \\
\hline Yes & $1(2 \%)$ & $1(2 \%)$ & $1(2 \%)$ & \\
\hline No & $49(98 \%)$ & $44(98 \%)$ & $45(98 \%)$ & \\
\hline \multicolumn{4}{|l|}{ Fetal infections } & \multirow[t]{3}{*}{0.781} \\
\hline Yes & $5(10 \%)$ & $3(7 \%)$ & $3(7 \%)$ & \\
\hline No & $45(90 \%)$ & $42(93 \%)$ & $42(93 \%)$ & \\
\hline Weight (gm) - Mean (SD) & $3251(60)$ & $3259(63)$ & $3247(67)$ & $0.935^{(\mathrm{kw})}$ \\
\hline Temperature - Mean (SD) & $36.14(0.08)$ & $36.3(0.73)$ & $36.22(0.08)$ & $0.347^{(\mathrm{kw})}$ \\
\hline Cord Blood RBC - Mean (SD) & $4.57 \times 10^{6}(0.07)$ & $4.45 \times 10^{6}(0.08)$ & $4.61 \times 10^{6}(0.07)$ & $0.489^{(k w)}$ \\
\hline Cord Blood TSB - Mean (SD) & $1.52(0.1)$ & $1.45(0.12)$ & $1.52(0.16)$ & $0.57^{(\mathrm{kw})}$ \\
\hline
\end{tabular}

Unadjusted linear regression of total serum bilirubin amount with umbilical cord clamping time revealed a negative but non-significant association between the two variables (Coef. -0.15 ; P-value $=0.19$ ). This is presented in Table 4 below.

Table 4: Simple regression of the total serum bilirubin level by time of cord clamping of newborns born at TASH from a period of October 2019 - January 2020.

\begin{tabular}{lllll|l}
\hline TSB & Coef. & Std. Err. & t & \multicolumn{1}{l}{ P-value } & \multicolumn{1}{c}{$\mathrm{R}^{2}$} \\
\hline Time of cord clamping & -0.1483 & 0.1134 & -1.31 & 0.193 & \multirow{2}{*}{0.012} \\
\cline { 1 - 5 } _const & 1.911158 & .2428028 & 7.87 & 0.000 & \\
\hline
\end{tabular}

Multiple regression analysis revealed that bilirubin nomogram high-risk zone $(p<0.001)$ and cord blood bilirubin $(p<0.05)$ were significant predictors of serum bilirubin levels within 24 hours of birth and delaying cord clamping time to three-minute shows no significant association with the total serum bilirubin (TSB) levels within 24 hours of birth. 
Table 5: Multivariate regressions of total serum bilirubin level as affected by different predictors within 24 hours of birth among neonates born at TASH from a period of October 2019 - January 2020.

\begin{tabular}{|c|c|c|c|c|c|}
\hline \multicolumn{6}{|c|}{$R^{2}=0.3614$, Adjusted $R^{2}=0.3422$} \\
\hline Total serum Bilirubin level & Coef. & Std. Err. & $\mathrm{t}$ & P-value & Beta \\
\hline Delayed cord clamping & -0.0695 & 0.162 & -0.43 & 0.669 & -0.0298 \\
\hline Cord blood RBC & -0.1056 & 0.14 & -0.75 & 0.453 & -0.0524 \\
\hline Cord blood TSB & 0.2395 & 0.0925 & 2.59 & 0.011 & 0.1962 \\
\hline Nomogram high-risk zone & 6.2554 & 0.976 & 6.41 & $<0.001$ & 0.4859 \\
\hline _Const. & 1.7133 & 0.663 & 2.58 & 0.011 & -- \\
\hline
\end{tabular}

Abbreviations: RBC, red blood cell; TSB, total serum bilirubin

\section{Discussion}

This study tested the hypothesis that delayed and immediate umbilical cord clamping would have a similar predictive effect on TSB levels of healthy full-term newborns within 24 hours of birth. Our findings indicate that the time of umbilical cord clamping did not affect TSB levels that is measured at 24 hours after birth. This finding implies supports that the arguments on the safety of DCC unlike previous findings reporting delayed time of cord clamping is associated with increased serum bilirubin and related jaundice and its complications. ${ }^{14-17}$

A study conducted on effects of DCC on residual placental blood volume, hemoglobin and bilirubin levels in term infants indicated that there was a significant increase in the number of neonates required phototherapy when there was a delay in the umbilical cord clamping time to 3 minutes. ${ }^{11}$ On the contrary, our finding indicates that there were only three newborns (one in each arm) that develops jaundice requiring phototherapy. This may be attributed to the fact that in our study serum bilirubin levels measured within 24 hours after birth and that can minimize the chance of capturing the late (48-72 hours) elevations in serum bilirubin levels.

Elevated cord blood RBC level or polycythemia was found to be a significant predictor of elevation of TSB around the early hours (24-72 hours) of life. ${ }^{12,18}$ Further, cord blood TSB was also significantly associated with serum bilirubin levels after 24 hours of birth. On the other hand, the cord blood RBC was not different from the normal range in any of the groups, which implies that an umbilical cord clamping time should not be considered as something detrimental to newborns health.

In our study, Bilirubin Nomogram high-risk zone was strongly associated with TSB levels within 24 hours of birth. This is different from what was reported previously by Judith et $a l^{11}$ that found an insignificant relationship between these variables. This discrepancy could be attributed to the fact that our study had only one participant in the high-risk zone. Nevertheless, using Bilirubin Nomogram is believed to be a gold-standard measurement to clearly predict, monitor and manage elevated bilirubin level at the early hours of the newborn before jaundice and further complications developed. ${ }^{19,20}$ 


\section{Conclusion}

This research finding found out and concluded that time of umbilical cord clamping will have no significant relationship with the total serum bilirubin levels of neonates at least within 24 hours of birth. In addition, delaying an umbilical cord clamping time to three minutes had no effect on elevating the total serum bilirubin levels of neonates that can put them in danger of jaundice requiring phototherapy and it can be considered as an easy and safe procedure that have a multifaceted importance for the newborns.

\section{Abbreviations}




\begin{tabular}{ll} 
AAU & Addis Ababa University \\
\hline ACOG & American College of Obstetrics and Gynecology \\
\hline BEMONC & Basic and Emergency Obstetrics and Newborn care \\
\hline DCC & Delayed Cord Clamping \\
\hline ECC & Early Cord Clamping \\
\hline EMwA & Ethiopian Midwives association \\
\hline EPS & Ethiopian Pediatric Society \\
\hline ESOG & Ethiopian Society of Obstetrics and Gynecology \\
\hline EWEC & Every Woman Every Child \\
\hline ICC & Immediate Cord Clamping \\
\hline IRB & Institutional Review Board \\
\hline LMP & Last Day of Menstrual Period \\
\hline LMIC & Low and Middle-Income Countries \\
\hline MOH & Ministry of Health (Ethiopia) \\
\hline RBCs & Red Blood Cells \\
\hline RCOG & Royal College of Obstetrics and Gynecology \\
\hline SDG & Sustainable development goal \\
\hline SPH & School of Public Health \\
\hline SVD & Spontaneous Vaginal Delivery \\
\hline TCC & Time of Cord Clamping \\
\hline TORCH & Toxoplasmosis, Other viruses, Rubella virus, Cytomegalo virus \& Herpes \\
\hline TSB & Total Serum Bilirubin \\
\hline UNICEF & United Nations Children's Fund \\
\hline WHO & World Health Organization \\
\hline
\end{tabular}

\section{Declarations}

\section{Competing interests:}

The authors declare no competing interests. 


\section{References}

1. UNICEF. Press-releases on global child birth report. Press release -child birth report January 1, 2018; https://www.unicef.org/media/media_102362.html. Accessed September 2018, January 2018.

2. EPHI, FMOH. Ethiopian Emergency Obstetric and Newborn Care (EmONC) Assessment. Ethiopian public health institute; September 20172016.

3. WHO. Guideline: Delayed umbilical cord clamping for improved maternal and infant health and nutrition outcomes. World Health Organization; 2014.

4. Eichenbaum-Pikser G, Zasloff J. Delayed clamping of the umbilical cord: a review with implications for practice. Journal of midwifery \& women's health. 2009;54(4):321-326.

5. Drummond G, Kappas A. Chemoprevention of severe neonatal hyperbilirubinemia. Seminars in perinatology. 2004;28(5):365-368.

6. Kamisako T, Kobayashi Y, Takeuchi K, et al. Recent advances in bilirubin metabolism research: the molecular mechanism of hepatocyte bilirubin transport and its clinical relevance. Journal of gastroenterology. 2000;35(9):659-664.

7. Watson R. Hyperbilirubinemia. Critical care nursing clinics of North America. 2009;21(1):97-120, vii.

8. Greco C, Arnolda G, Boo N, et al. Neonatal Jaundice in Low- and Middle-Income Countries: Lessons and Future Directions from the 2015 Don Ostrow Trieste Yellow Retreat. Neonatology. 2016;110(3):172-180.

9. Kassa RT, Gudeta H, Assen ZM, Demlew TM, Teshome GS. Neonatal Hyperbilirubinemia: Magnitude and Associated Etiologic Factors among Neonates Admitted at Tikur Anbessa Specialized Hospital, Ethiopia. Journal of Pregnancy and Child Health. 2018;5(4):4.

10. Cernadas JMC. Timing of umbilical cord clamping of term infants. Arch Argent Pediatr. 2017;115(2):1-7.

11. Mercer JS, Erickson-Owens DA, Collins J, Barcelos MO, Parker AB, Padbury JF. Effects of delayed cord clamping on residual placental blood volume, hemoglobin and bilirubin levels in term infants: a randomized controlled trial. Journal of Perinatology. 2017;37(3):260-264.

12. Andersson $O$, Hellström-Westas L, Andersson D, Domellöf M. Effect of delayed versus early umbilical cord clamping on neonatal outcomes and iron status at 4 months: a randomised controlled trial. BMJ. 2011;343(1):12.

13. Purisch SE, Ananth CV, Arditi B, et al. Effect of Delayed vs Immediate Umbilical Cord Clamping on Maternal Blood Loss in Term Cesarean Delivery: A Randomized Clinical Trial. Jama. 2019;322(19):1869-1876.

14. Anna Locatelli, Consonni S, Ghidini A. Approach to Decreasing Complications of Prematurity. Obstet Gynecol Clin N Am. 2015;1(1):1-20.

15. Duley L, Batey N. Optimal timing of umbilical cord clamping for term and preterm babies. Early human development. 2013;89:905-908. 
16. McDonald S, Middleton P. Effect of timing of umbilical cord clamping of term infants on maternal and neonatal outcomes. cochrane library. 2008;2008(2):1-29.

17. Saba K, Majeed T, Bukhari MH. Early versus Delayed Umbilical Cord Clamping Leads to Neonatal Anemia. ANNALS. 2012;18(3):309-315.

18. Rincon $D$, Foguet $A$, Rojas $M$, et al. [Time of cord clamping and neonatal complications, a prospective study]. Anales de pediatria (Barcelona, Spain : 2003). 2014;81(3):142-148.

19. Bhutani VK. Bilirubin nomogram, a prediction tool or natural history profile? Indian pediatrics. 2013;50(4):365-366.

20. Yu ZB, Han SP, Chen C. Bilirubin nomograms for identification of neonatal hyperbilirubinemia in healthy term and late-preterm infants: a systematic review and meta-analysis. World journal of pediatrics : WJP. 2014;10(3):211-218.

\section{Figures}

\section{Total Screening}

(674)

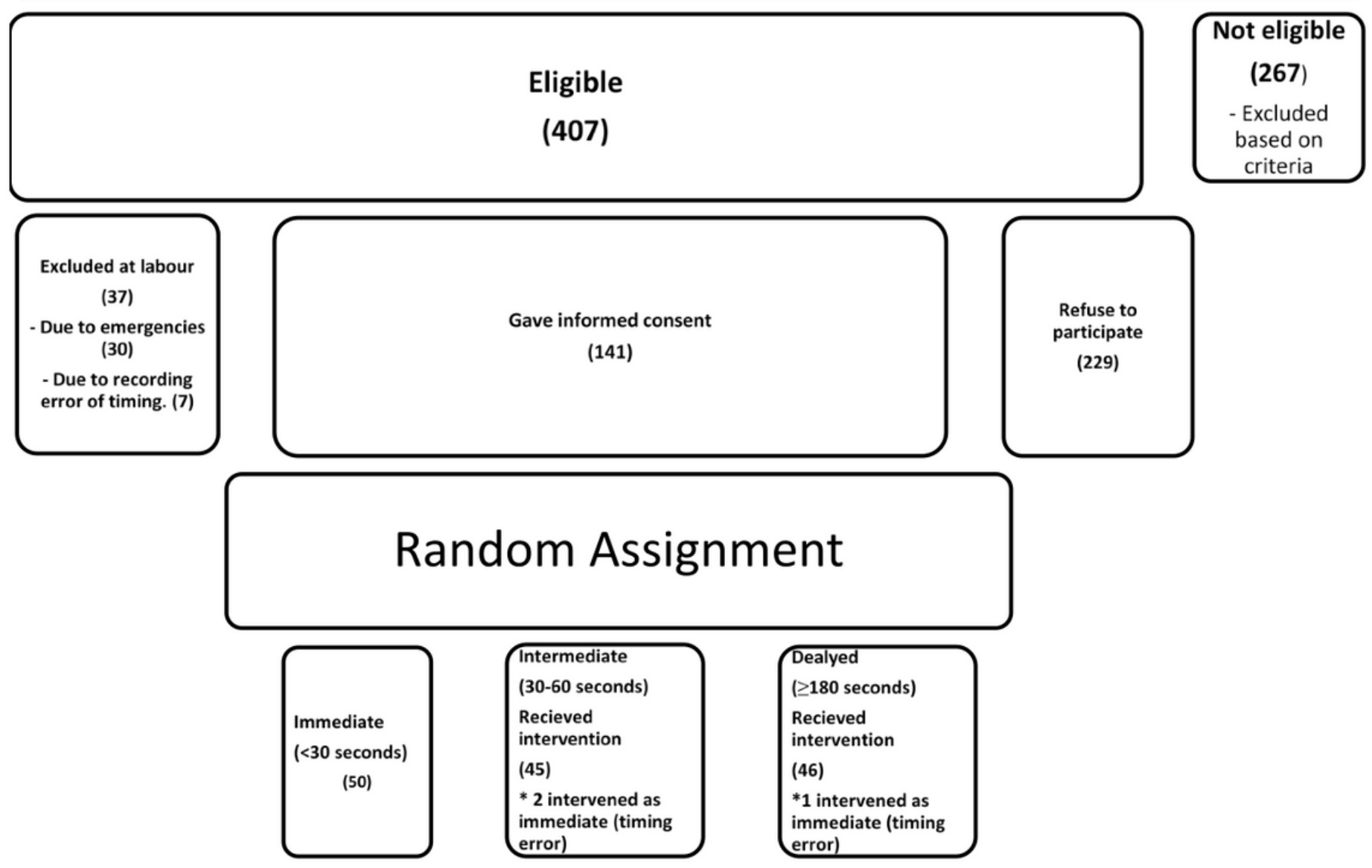


Sampling technique of study participants from mothers came for child birth at TASH from October 2019 - January 2020.

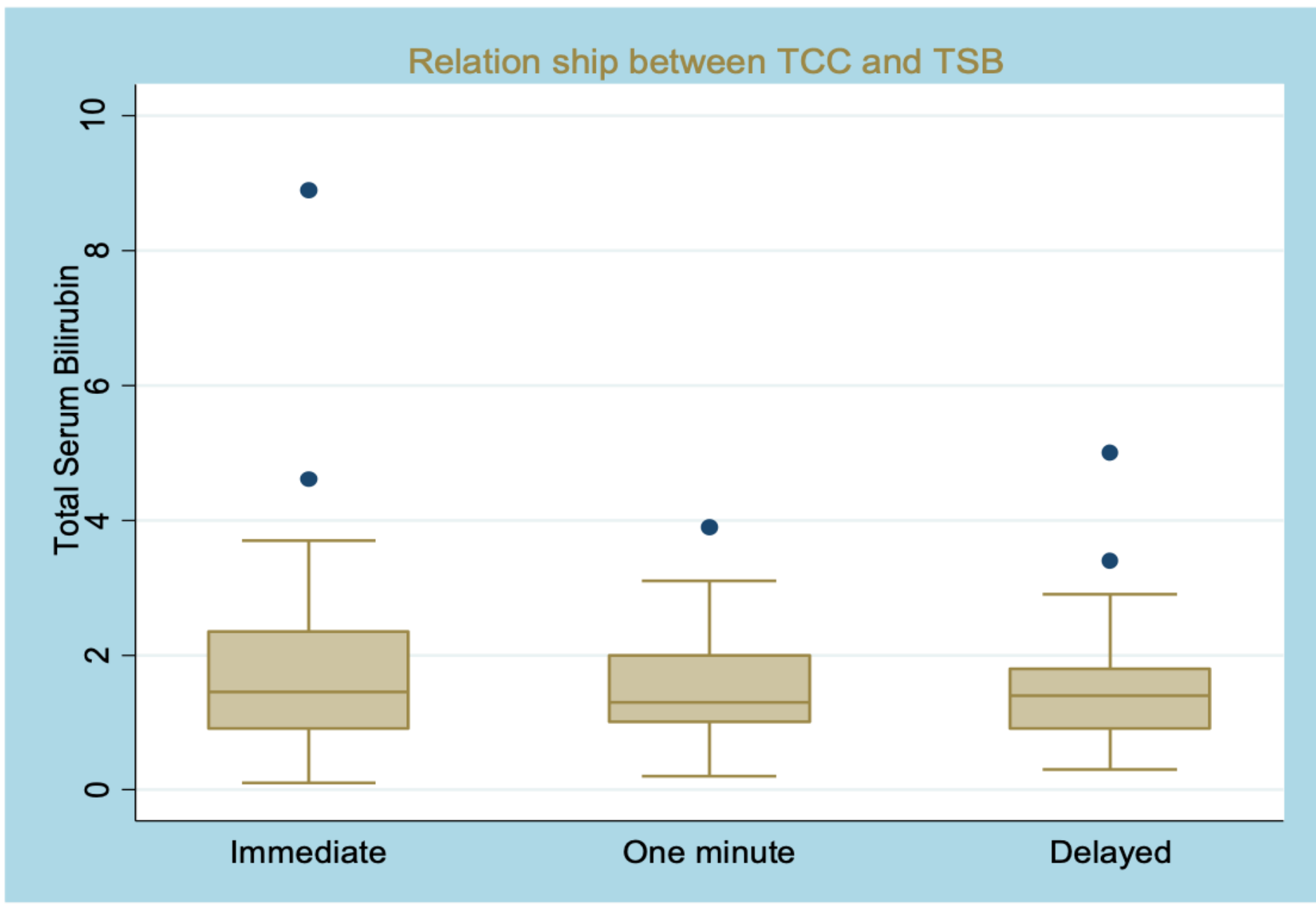

Figure 2

Relationship between time of cord clamping and total serum bilirubin level at the age of 24 hours of birth among neonates born at TASH from a period of October 2019 - January 2020. 